\title{
Derivation of statistical energy analysis from radiative exchanges
}

\author{
A. LE BOT \\ Laboratoire de Tribologie et Dynamique des Systèmes CNRS \\ École centrale de Lyon, FRANCE alain.le-bot@ec-lyon.fr
}

\begin{abstract}
This paper is concerned with the derivation of SEA equations from structural ray equations. Rays are assumed to be uncorrelated leading to the additivity of energy. Inside all subsystems, the energy density is the sum of a direct field from driving forces, a reflected field from the boundary and a transmitted field from adjacent subsystems. Assuming a "rain-on-theroof" excitation and a compact shape for subsystems, actual and fictitious sources on the boundary are found to be constant. Furthermore, if the attenuation of rays during a mean free path (normalized attenuation factor) is light, the field becomes diffuse i.e. homogeneous and isotropic. The net exchanged power between two adjacent subsystems is then proportional to the difference of energy densities and therefore, to the difference of modal energies. The derived proportionality coefficient is consistent with the well-known formula for coupling loss factor in terms of transmission factors. These results are illustrated by a numerical simulation for a multi-plate system. Finally, the validity domains of SEA and the ray theory are discussed and particularly the diffuse field assumption.
\end{abstract}

\section{Introduction}

Several approaches have been adopted to derive SEA equations. The historical approach is based on the study of a pair of coupled resonators randomly excited for which the famous proportionality between the energy flow and the difference of vibrational energies has been first published $[1,2]$. The generalization to two sets of oscillators in interaction requires four further assumptions. Firstly, oscillators within the same set do not exchange energy and thus, the power exchanged between the two sets is the sum of exchanged powers of each pair of resonators, one in each set. Secondly, the excitation forces are uncorrelated and, in the case of continuous structures, the force field is assumed to be "rain-on-the-roof" that is the random force is a white noise at any point and also that the field is spatially $\delta$-correlated. Thirdly, the coupling between the two sets is weak. Finally, the vibrational energy is equally shared by oscillators of the same set. This equirepartition of energy turns out to be the key concept of SEA and it means that each set of oscillators is in thermal equilibrium. Under all these assumptions, the exchanged power is proportional to the difference of modal energies of the sets of oscillators [3]. The modal approach of SEA may be compared with the statistical approach of molecular physics and until today, it has been an attractive method leading to new theoretical results $[4,5]$.

At the same time, the wave approach has been developed on the concept of travelling waves rather than modes $[6,7]$. All previously enunciated assumptions have their equivalent in the wave 
approach although the debate about their strict equivalence seems not to be closed. The two main assumptions of the wave approach are that waves are uncorrelated and that the vibrational field is diffuse. This last assumption is equivalent to the equirepartition of energy in the modal approach. This equivalence is highlighted by the proportionality between modal energy and energy density (or intensity) stemming from the proportionality of modal density and length, surface or volume of a subsystem. The wave approach has proved its efficiency to solve some questions where the modal approach was difficult to apply. This is the case for the relationships giving the coupling loss factor in terms of the transmission efficiency at junctions [8, 9]. Extensive reviews of SEA with more complete bibliographies are available in the standard books $[10,11,12]$. An historical review and a criticism approach of SEA may be found in Ref. [13].

Adopting the spirit of SEA, some generalizations have been proposed for non diffuse fields. In the wave intensity analysis [14], the assumption of isotropy is relaxed whereas in the vibrational conductivity approach [15] homogeneity and isotropy are both relaxed. Another method is proposed in Ref. [16] to relax homogeneity and isotropy. Based on the assumption that all rays are uncorrelated, the method consists to write the power balance on incoming and outcoming waves on the boundary. Introducing a fictitious source layer on the boundary, the power of these sources is governed by an integral equation (radiative transfer equation) exactly as it is in the view factor method in thermics [17] or in the radiosity method in acoustics [18]. The method applies from diffuse to largely non-diffuse field in assembled plates. A last generalization of SEA is proposed by Maxit and Guyader [19] when the equirepartition of modal energy does not hold. The method named the statistical modal energy distribution analysis is based on the energy balance of individual modes instead of sets of modes as in SEA.

The question of the consistency of these generalizations with SEA itself naturally arises. Any candidate for generalizing SEA must embody SEA as a particular case. If the answer is somewhat obvious for the wave intensity analysis [14], the vibrational conductivity [20] and the statistical modal energy distribution analysis [19], it is not for radiative exchanges. There are several reasons to examine the derivation of SEA from radiative exchanges beyond the mathematical requirement of consistency. Firstly, since radiative exchanges are formally equivalent to the ray-tracing technique [21], it highlights the link between geometrical acoustics including structural rays and SEA. Secondly, SEA may be viewed as the theory of thermal equilibrium of mechanical vibrations whereas the theory of rays could rather be compared with the kinetic theory of gas so that it is valid beyond equilibrium. To derive SEA from radiative transfer is thus a way to investigate the transition from non-equilibrium state to equilibrium state. This question has some immediate consequences of practical interest for SEA. The stakes are to be able to recognize the situations where equilibrium is reached i.e. when diffuse field is established. This rises the important question of the validity of SEA.

The purpose of the present paper is therefore to derive the basic relationships of SEA by the wave approach of Ref. [16]. In particular, this paper focuses on the net exchanged power between two subsystems with a common boundary. Starting from radiative exchanges of uncorrelated rays, it is found that the diffuse field assumption leads to the expected equation. The obtained proportionality coefficient is consistent with the well-known coupling loss factor for adjacent subsystems of the traditional wave approach of SEA.

The outline of this paper is as follows. In Section 2, the basic integral equations of uncorrelated rays are given for any fields, diffuse or not diffuse. In Section 3, the equations for energy, intensity and exchanged power are investigated for the case of constant domain sources and boundary sources. The diffuse field is obtained in Section 4 when the direct field is negligible and the wellknown equations of SEA are finally derived. A numerical simulation is proposed in Section 5 and 
finally, a discussion on the validity of both methods is done in Section 6 .

\section{Equations of uncorrelated ray fields}

In the framework of SEA, vibrating systems are divided into several subsystems. In general, a subsystem is defined as a set of modes of the same structural component for which the equirepartition of energy applies and which are lightly coupled with other subsystems. As present paper focuses on the case of assembled plates, the subsystems, noted $\Omega_{i}, i=1 \ldots n$ are the flexural, the longitudinal and the transverse modes of each plate. However, the demonstration can be straightforwardly generalized to the case of beam networks, or adjacent rooms provided that the solid angle of space and the law of decrease of energy and some other minor modifications have been achieved. The important assumption of the demonstration is that subsystems can only exchange energy with other subsystems through their boundaries. Thus, radiation of sound, structural response or the coupling of plates with their reinforcing beams are excluded of the present demonstration.

The main assumption of the wave approach of SEA is that fields are diffuse in all subsystems. The conditions which lead to diffuse field are studied by the mathematical theory of billiards. It is found that some shapes, the so-called "mixing" billiards, naturally give a diffuse field by mixing the rays after several reflections on boundaries [22]. Uncorrelation of rays and Lambert's law for reflection are then two statistical consequences of mixing of rays even if individual rays have a deterministic phase and specularly reflect on the boundaries. Uncorrelation of rays also implies the additivity of energy of individual rays.

We then start from a ray theory whose primary variables are energy and intensity and which admits the cosine Lambert's law for the energy reflected on boundaries. This is exactly the assumptions of the so-called "factor view method" or "standard method" in thermics. An adaptation of this method to the case of assembled plates coupled by their edges is achieved in Ref. [16]. In the rest of this section, all basic relationships and the theoretical material necessary for this paper is introduced without full demonstrations.

Let us consider a stationary point source of unit power in a two-dimensional system. The energy density at a distance $R$ is $e^{-m R} / 2 \pi c R$ where $m$ is the attenuation factor responsible of dissipation during propagation and $c$ is the group speed. In presence of several sources whose power density is noted $\rho_{i}$, the energy density $W_{i}$ at any point $\mathbf{r}$ in the subsystem $\Omega_{i}$, is not only the sum of direct fields $\rho_{i} \exp ^{-m R} / 2 \pi c R$ but also reflected and transmitted fields introduced by some fictitious sources $\sigma_{i}$ located over the boundary $\Gamma_{i}$,

$$
W_{i}(\mathbf{r})=\int_{\Omega_{i}} \rho_{i}(\mathbf{s}) \frac{\exp ^{-m R}}{2 \pi c R} \mathrm{~d} \Omega_{\mathbf{s}}+\int_{\Gamma_{i}} \sigma_{i}(\mathbf{p}) \cos \theta \frac{\exp ^{-m R}}{2 \pi c R} \mathrm{~d} \Gamma_{\mathbf{p}},
$$

where $\theta$ is the emission angle at point $\mathbf{p}$ measured with the normal of the boundary and $R=|\mathbf{s}-\mathbf{r}|$ or $|\mathbf{p}-\mathbf{r}|$ is the source-receiver distance. As it was previously claimed, the boundary sources $\sigma_{i}$ radiate energy in accordance with the cosine Lambert's law.

When evaluating the energy density at any point $\mathbf{p}$ on the boundary $\Gamma_{i}$, an additional term appears,

$$
W_{i}(\mathbf{p})=\int_{\Omega_{i}} \rho_{i}(\mathbf{s}) \frac{\exp ^{-m R}}{2 \pi c R} \mathrm{~d} \Omega_{\mathbf{s}}+\int_{\Gamma_{i}} \sigma_{i}(\mathbf{q}) \cos \theta \frac{\exp ^{-m R}}{2 \pi c R} \mathrm{~d} \Gamma_{\mathbf{q}}+\frac{\sigma_{i}(\mathbf{p})}{2 c} .
$$

The presence of the last term will be clarified in the next section. 


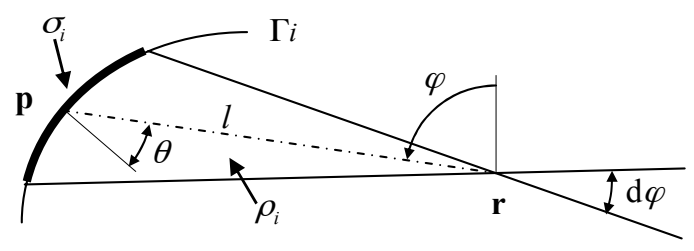

Figure 1: Radiative intensity

A further concept useful for our purpose is the so-called "radiative intensity" or "specific intensity". This is the power per unit angle and unit length normal to the ray. Consider a point $\mathbf{r} \in \Omega_{i}$ and an infinitesimal angle $\mathrm{d} \varphi$ (Fig. 1). The power per unit length passing through that point is the sum of contributions of all sources $\rho_{i}$ located inside the cone of vertex $\mathbf{r}$ and angle $\mathrm{d} \varphi$ and the sources $\sigma_{i}$ on the part of boundary inside this cone.

$$
\mathrm{d} P=\int \rho_{i} \frac{\mathrm{e}^{-m R}}{2 \pi R} \mathrm{~d} \Omega+\sigma_{i} \cos \theta \frac{\mathrm{e}^{-m l}}{2 \pi l} \mathrm{~d} \Gamma,
$$

where the first integral is performed over the cone, $\mathrm{d} \Gamma$ is the piece of boundary inside the cone and $l(\mathbf{r}, \varphi)$ is the distance from $\mathbf{r}$ to the boundary in direction $\varphi$ (Fig. 1). Since $\mathrm{d} \Omega=R \mathrm{~d} \varphi \mathrm{d} R$ and $\mathrm{d} \Gamma=l \mathrm{~d} \varphi / \cos \theta$, it yields,

$$
\mathrm{d} P=\int \rho_{i} \frac{\mathrm{e}^{-m R}}{2 \pi R} R \mathrm{~d} \varphi \mathrm{d} R+\sigma_{i} \cos \theta \frac{\mathrm{e}^{-m l}}{2 \pi l} \frac{l \mathrm{~d} \varphi}{\cos \theta} .
$$

Therefore, the radiative intensity $I_{i}=\mathrm{d} P / \mathrm{d} \varphi$ is,

$$
I_{i}(\mathbf{r}, \varphi)=\int_{0}^{l(\mathbf{r}, \varphi)} \rho_{i}(\mathbf{s}) \frac{\mathrm{e}^{-m R}}{2 \pi} \mathrm{d} R+\frac{\sigma_{i}(\mathbf{p})}{2 \pi} \mathrm{e}^{-m l(\mathbf{r}, \varphi)},
$$

where $\mathbf{p}$ is the first point on the boundary encountered from $\mathbf{r}$ in direction $-\varphi$.

At the interface of length $L$ between two subsystems $j$ and $i$, a part of the incident power is transmitted while the other part is reflected. Introducing the transmission efficiency $\tau_{j i}(\varphi)$ depending on the incidence angle $\varphi$ and which is defined as the ratio of the transmitted power over the incident power, the power transmitted from subsystem $j$ to subsystem $i$ is,

$$
P_{j \rightarrow i}=L \int_{-\pi / 2}^{\pi / 2} \tau_{j i}(\varphi) I_{j}(\mathbf{p}, \varphi) \cos \varphi \mathrm{d} \varphi,
$$

where the integration is performed over all possible incidence angles. In this expression, $I_{i} \cos \varphi$ is the incident power per unit length from direction $\varphi, \tau_{j i}(\varphi) I_{i} \cos \varphi$ is the part of this power which is transmitted and $P_{j \rightarrow i}$ is the total transmitted power from all directions.

The last question which has not yet been tackled is how to determine the fictitious source strength $\sigma_{i}$. Consider a point $\mathbf{p} \in \Gamma_{i}$ on the boundary. The incident power stemming from $\Omega_{i}$ 
is $\int I_{i} \cos \varphi \mathrm{d} \varphi$. So, if the boundary has an absorption coefficient $\alpha$ and therefore, a reflection coefficient $1-\alpha$, the energy balance reads,

$$
\frac{\sigma_{i}(\mathbf{p})}{\pi}=(1-\alpha) \int_{-\pi / 2}^{\pi / 2} I_{i}(\mathbf{p}, \varphi) \cos \varphi \mathrm{d} \varphi
$$

where the left-hand side is the power per unit length of boundary radiated by the fictitious source $\sigma_{i}$. Substitution of Eq. (5) into Eq. (7) and a change of variable leads to,

$$
\frac{1}{\pi} \sigma_{i}(\mathbf{p})=(1-\alpha)\left(\int_{\Omega_{i}} \rho_{i}(\mathbf{s}) \frac{\mathrm{e}^{-m R}}{2 \pi R} \cos \varphi \mathrm{d} \Omega_{\mathbf{s}}+\int_{\Gamma_{i}} \sigma_{i}(\mathbf{q}) \cos \theta \frac{\mathrm{e}^{-m R}}{2 \pi R} \cos \varphi \mathrm{d} \Gamma_{\mathbf{q}}\right) .
$$

This Fredholm equation of second kind gives the unknown $\sigma_{i}$ at point $\mathbf{p}$ in terms of all structural sources $\rho_{i}$ and other boundary sources $\sigma_{i}(\mathbf{q})$. It is similar to the radiative transfer equation in thermics which gives the radiative intensity in terms of power incident from all other directions.

Now, if the point $\mathbf{p}$ is located on the common edge of several subsystems, the radiated power per unit length $\sigma_{i} / \pi$ stems from all other adjacent subsystems and therefore the power balance reads,

$$
\frac{\sigma_{i}(\mathbf{p})}{\pi}=\sum_{j} \frac{\mathrm{d} P_{j \rightarrow i}}{\mathrm{~d} L} .
$$

Successive substitution of Eqs. (6) and (5) into Eq. (9) leads to,

$$
\frac{1}{\pi} \sigma_{i}(\mathbf{p})=\sum_{j} \int_{\Omega_{j}} \tau_{j i}(\varphi) \rho_{j}(\mathbf{s}) \frac{\mathrm{e}^{-m R}}{2 \pi R} \cos \varphi \mathrm{d} \Omega_{\mathbf{s}}+\int_{\Gamma_{j}} \tau_{j i}(\varphi) \sigma_{j}(\mathbf{q}) \cos \theta \frac{\mathrm{e}^{-m R}}{2 \pi R} \cos \varphi \mathrm{d} \Gamma_{\mathbf{q}} .
$$

Once again, $\sigma_{i}(\mathbf{p})$ is given in terms of all other unknowns $\sigma_{j}(\mathbf{q})$.

\section{$3 \quad$ Energy, intensity and power with constant sources}

A rain-on-the-roof excitation means that the subsystem is entirely covered by some random driving forces. These forces are spatially $\delta$-correlated and have a power spectrum density constant in frequency (white noise) and in space (uniformity). In the framework of the theory of the previous section, rain-on-the-roof excitation means that $\rho_{i}$ is constant over the subsystem $\Omega_{i}$.

Rain-on-the-roof excitation is generally not sufficient to ensure a diffuse field. Mixing of rays requires a large number of reflections only possible when their energy does not decrease too fast. Light damping loss factors and coupling loss factors is therefore a necessary condition. But in addition, a certain geometrical condition on the shape of subsystems is necessary. We first assume that this condition is satisfied and leads a constant value of the fictitious sources $\sigma_{i}$. After the expressions for energy and intensity have been derived, it will be possible to find this condition and therefore to define the validity domain of these expressions.

Assuming that $\rho_{i}$ and $\sigma_{i}$ are constant and neglecting the attenuation factor $m$ (light damping), the energy density is from Eq. (1),

$$
W_{i}(\mathbf{r})=\rho_{i} \int_{\Omega_{i}} \frac{\mathrm{d} \Omega}{2 \pi c R}+\sigma_{i} \int_{\Gamma_{i}} \frac{\cos \theta}{2 \pi c R} \mathrm{~d} \Gamma,
$$


at any point $\mathbf{r}$ inside $\Omega_{i}$, and from Eq. (2),

$$
W_{i}(\mathbf{p})=\rho_{i} \int_{\Omega_{i}} \frac{\mathrm{d} \Omega}{2 \pi c R}+\sigma_{i} \int_{\Gamma_{i}} \frac{\cos \theta}{2 \pi c R} \mathrm{~d} \Gamma+\frac{\sigma_{i}}{2 c},
$$

at any point $\mathbf{p}$ on the boundary $\Gamma_{i}$. In Eqs. (11) and (12) appear two integrals,

$$
\begin{gathered}
A(\mathbf{r})=\int_{\Omega} \frac{\mathrm{d} \Omega}{2 \pi R}, \\
B(\mathbf{r})=\int_{\Omega} \frac{\cos \theta}{2 \pi R} \mathrm{~d} \Gamma,
\end{gathered}
$$

which are functions of the receiver point $\mathbf{r}$.

By expanding $\mathrm{d} \Omega=R \mathrm{~d} R \mathrm{~d} \varphi$ in polar coordinates, the first integral becomes,

$$
A(\mathbf{r})=\int_{0}^{2 \pi} \int_{0}^{l(\mathbf{r}, \varphi)} \frac{R \mathrm{~d} R \mathrm{~d} \varphi}{2 \pi R}=\frac{1}{2 \pi} \int_{0}^{2 \pi} l(\mathbf{r}, \varphi) \mathrm{d} \varphi,
$$

where $l(\mathbf{r}, \varphi)$ is the distance from $\mathbf{r}$ to $\Gamma$ in direction $\varphi . l(\mathbf{r}, \varphi)+l(\mathbf{r},-\varphi)$ is the boundary to boundary distance and therefore $A(\mathbf{r})$ is half the mean boundary to boundary distance viewed from $\mathbf{r}$. This average distance is expected to be close to the the mean free path $\bar{l}$. The definition of the mean free path is,

$$
\bar{l}=\frac{1}{P} \int_{\Gamma} \mathrm{d} \Gamma_{\mathbf{p}} \int_{-\pi / 2}^{\pi / 2} l(\mathbf{p}, \varphi) \frac{\cos \varphi}{2} \mathrm{~d} \varphi=\pi \frac{S}{P},
$$

where $S$ is the area of the domain $\Omega$ and $P$ is the perimeter of the boundary $\Gamma$. This last equality is the classical expression for mean free path in bi-dimensional systems. It is obtained from the first equality after few algebra. A difference appears between the integral involved in Eq. (15) and those of Eq. (16). The function $\cos \varphi / 2$ is the probability density for a ray to have an incidence $\varphi$. Thus, let us introduce the function $\zeta$ defined as the ratio $\zeta=\int l d \varphi / \pi \bar{l}$, that is,

$$
A(\mathbf{r})=\frac{\bar{l}}{2} \zeta(\mathbf{r}) .
$$

$\zeta$ is a dimensionless function confined in the neighbourhood of unity. When $\mathbf{r} \rightarrow \mathbf{p} \in \Gamma, l(\mathbf{p}, \varphi)$ is the boundary to boundary distance for inward direction $\varphi$ whereas $l(\mathbf{p},-\varphi)=0 . A(\mathbf{r})$ is therefore a continuous function when $\mathbf{r}$ goes to $\mathbf{p} \in \Gamma$.

For the second integral, consider the change of variable $\mathrm{d} \Gamma=R \mathrm{~d} \varphi / \cos \theta$ where, as usual, $\mathrm{d} \Gamma$ is the measure of boundary length and $R, \varphi$ are the polar coordinates of the current point of boundary in the framework centred in $\mathbf{r} \in \Omega$ (Fig. 2a). Any interior point $\mathbf{r} \in \Omega$ is surrounded by $\Gamma$ and therefore $\varphi$ runs from $-\pi$ to $\pi$ (Fig. $2 \mathrm{~b}$ ),

$$
B(\mathbf{r})=\int_{-\pi}^{\pi} \frac{\mathrm{d} \varphi}{2 \pi}=1 .
$$

But, for a boundary point $\mathbf{p} \in \Gamma, \varphi$ is limited to the range $-\pi / 2, \pi / 2$ (Fig. $2 \mathrm{c}$ ),

$$
B(\mathbf{p})=\int_{-\pi / 2}^{\pi / 2} \frac{\mathrm{d} \varphi}{2 \pi}=\frac{1}{2} .
$$


(a)

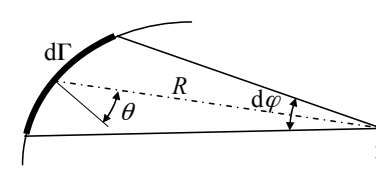

(b)

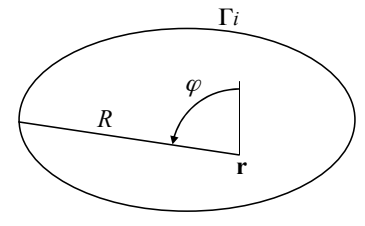

(c)

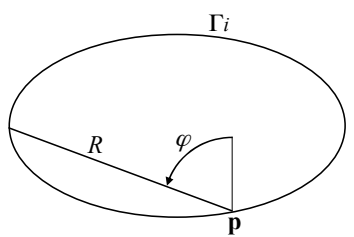

Figure 2: Polar coordinates centred in $\mathbf{r}$. (a), Relationship between the boundary length $\mathrm{d} \Gamma$ and $\mathrm{d} \varphi$. (b), for $\mathbf{r} \in \Omega, \varphi$ runs from $-\pi$ to $\pi$. (c), for $\mathbf{p} \in \Gamma, \varphi$ runs from $-\pi / 2$ to $\pi / 2$.

$B(\mathbf{r})$ is then a discontinuous function when $\mathbf{r}$ goes to $\mathbf{p} \in \Gamma$.

With these results, Eqs. (11) and (12) become,

$$
\begin{aligned}
W_{i}(\mathbf{r}) & =\rho_{i} \frac{\bar{l}}{2 c} \zeta+\frac{\sigma_{i}}{c}, \\
W_{i}(\mathbf{p}) & =\rho_{i} \frac{\bar{l}}{2 c} \zeta+\frac{\sigma_{i}}{2 c}+\frac{\sigma_{i}}{2 c} .
\end{aligned}
$$

$W_{i}$ is a continuous function, as should be all physical quantities. The explanation of the presence of the additional term in Eq. (2) is now apparent. This term compensates the discontinuity of the integral $\int \sigma \cos \theta \mathrm{e}^{-m R} / 2 \pi R \mathrm{~d} \Gamma$ when $\mathbf{r} \in \Omega$ goes to $\mathbf{p} \in \Gamma$, and then ensures the continuity of $W_{i}$.

For constant sources $\rho_{i}$ and $\sigma_{i}$, the radiative intensity is from Eq. (5),

$$
I_{i}(\mathbf{r}, \varphi)=\rho_{i} \int_{0}^{l(\mathbf{r}, \varphi)} \frac{\mathrm{d} R}{2 \pi}+\frac{\sigma_{i}}{2 \pi}=\frac{\rho_{i} l(\mathbf{r}, \varphi)}{2 \pi}+\frac{\sigma_{i}}{2 \pi} .
$$

The mean radiative intensity is,

$$
\bar{I}_{i}(\mathbf{r})=\frac{1}{2 \pi} \int_{0}^{2 \pi} I_{i}(\mathbf{r}, \varphi) \mathrm{d} \varphi=\frac{1}{2 \pi}\left(\rho_{i} \frac{1}{2 \pi} \int_{0}^{2 \pi} l(\mathbf{r}, \varphi) \mathrm{d} \varphi+\sigma_{i}\right),
$$

and therefore,

$$
\bar{I}_{i}(\mathbf{r})=\frac{1}{2 \pi}\left(\rho_{i} \frac{\bar{l}}{2} \zeta+\sigma_{i}\right)
$$

It results from Eq. (20),

$$
\bar{I}_{i}=\frac{c W_{i}}{2 \pi} .
$$

This is the equation usually written in the wave approach of SEA by assuming homogeneity and isotropy of field. It is found here that this equality applies in an average sense for constant $\rho_{i}$ and $\sigma_{i}$.

As the point $\mathbf{r}$ approaches the boundary $\Gamma$, it is necessary to distinguish the outward directions $|\varphi|>\pi / 2$ for which $l(\mathbf{p}, \varphi)$ is the boundary to boundary distance and inward directions $|\varphi|<\pi / 2$ 
for which $l(\mathbf{p}, \varphi)=0$. In the limit $\mathbf{r} \rightarrow \mathbf{p} \in \Gamma$,

$$
\begin{array}{r}
\bar{I}_{i}^{+}(\mathbf{p})=\frac{1}{\pi} \int_{|\varphi|>\pi / 2} I_{i}(\mathbf{p}, \varphi) \mathrm{d} \varphi=\frac{1}{2 \pi}\left(\rho_{i} \bar{l} \zeta+\sigma_{i}\right), \\
\bar{I}_{i}^{-}(\mathbf{p})=\frac{1}{\pi} \int_{|\varphi|<\pi / 2} I_{i}(\mathbf{p}, \varphi) \mathrm{d} \varphi=\frac{1}{2 \pi} \sigma_{i} .
\end{array}
$$

Indeed, the mean of these two quantities is the mean radiative intensity of Eq. (24). But Eqs. (26) and (27) show that near the boundary the radiative intensity is no longer equally shared among inward and outward components.

Finally, the transmitted power given by Eq. (6) is, with the additional condition of constant $\rho_{i}$ and $\sigma_{i}$,

$$
P_{j \rightarrow i}=L \int_{-\pi / 2}^{\pi / 2} \tau_{j i}(\varphi) \cos \varphi \mathrm{d} \varphi \times \bar{I}_{j}^{+},
$$

where the mean incident radiative intensity $\bar{I}_{j}^{+}$has been substituted for the exact radiative intensity $I_{j}(\mathbf{p})$. Introducing the average transmission efficiency,

$$
\bar{\tau}_{j i}=\frac{1}{2} \int_{-\pi / 2}^{\pi / 2} \tau_{j i}(\varphi) \cos \varphi \mathrm{d} \varphi
$$

the transmitted power is,

$$
P_{j \rightarrow i}=L \frac{\bar{\tau}_{j i}}{\pi}\left(\rho_{j} \bar{l} \zeta+\sigma_{j}\right)
$$

Eqs. (20), (22) and (30) constitute a set of equations on the energy quantities valid for constant sources. But we have not yet investigated the condition for $\sigma_{i}$ to be constant. $\sigma_{i}$ is related to domain sources $\rho_{i}$ by Eq. (8) for reflection and by Eq. (10) for transmission. Let consider Eq. (8) with constant $\rho_{i}$ and $\sigma_{i}$,

$$
\frac{\sigma_{i}}{\pi}=(1-\alpha) \mathrm{e}^{-m \bar{l}}\left(\rho_{i} \int_{\Omega_{i}} \frac{\cos \varphi}{2 \pi R} \mathrm{~d} \Omega+\sigma_{i} \int_{\Gamma_{i}} \frac{\cos \theta \cos \varphi}{2 \pi R} \mathrm{~d} \Gamma\right)
$$

where the term $\mathrm{e}^{-m R}$ has been substituted by its average value $\mathrm{e}^{-m \bar{l}}$. Once again, it appears as two integrals which only depend on the shape of $\Omega$,

$$
\begin{aligned}
C(\mathbf{p}) & =\int_{\Omega} \frac{\cos \varphi}{2 \pi R} \mathrm{~d} \Omega, \\
D(\mathbf{p}) & =\int_{\Gamma} \frac{\cos \theta \cos \varphi}{2 \pi R} \mathrm{~d} \Gamma .
\end{aligned}
$$

By the change of variable $\mathrm{d} \Gamma \cos \theta / R=\mathrm{d} \varphi$, the second integral is constant,

$$
D(\mathbf{p})=\int_{-\pi / 2}^{\pi / 2} \frac{\cos \varphi}{2 \pi} \mathrm{d} \varphi=\frac{1}{\pi} .
$$

For the first integral, the change of variable $\mathrm{d} \Omega=R \mathrm{~d} R \mathrm{~d} \varphi$ leads to,

$$
C(\mathbf{p})=\int_{-\pi / 2}^{\pi / 2} \frac{\cos \varphi}{2 \pi} \mathrm{d} \varphi \int_{0}^{l(\mathbf{p}, \varphi)} \mathrm{d} R=\frac{1}{2 \pi} \int_{-\pi / 2}^{\pi / 2} l(\mathbf{p}, \varphi) \cos \varphi \mathrm{d} \varphi .
$$


This is the average of the distances from $\mathbf{p}$ to other points of $\Gamma$ weighted by the function $\cos \varphi / 2$ and divided by $\pi$. From Eq. (34), Eq. (31) becomes,

$$
\frac{\sigma_{i}}{\pi}=\frac{1-\alpha \mathrm{e}^{-m \bar{l}}}{1-(1-\alpha) \mathrm{e}^{-m \bar{l}}} \rho_{i} C(\mathbf{p}) .
$$

The question of constancy of $\sigma_{i}$ is equivalent to the constancy of $C(\mathbf{p})$. By virtue of symmetry, $C(\mathbf{p})$ is constant for a disk and therefore $\sigma_{i}$ too (see Eq. (31)). When $\Omega$ is not a disk, $C(\mathbf{p})$ and therefore $\sigma_{i}$ are approximately constant when $\Omega$ has a compact shape in the sense that it has not a size much larger than the other ones. But, Eq. (36) rather shows the inverse. The only way for $\sigma_{i}$ to be constant is that $C(\mathbf{p})$ is constant and therefore that $\Omega$ has a compact shape.

By comparing Eq. (35) and Eq. (16), the mean free path is $\bar{l}=\pi \int C \mathrm{~d} \Gamma / P$ and therefore, the constancy of $C(\mathbf{p})$ reads,

Finally,

$$
C(\mathbf{p})=\frac{\bar{l}}{\pi} .
$$

$$
\sigma_{i}=\frac{1-\alpha \mathrm{e}^{-m \bar{l}}}{1-(1-\alpha) \mathrm{e}^{-m \bar{l}}} \rho_{i} \bar{l} .
$$

In summary, Eqs. (20), (22) and (30) respectively derived for energy, intensity and power apply when $\Omega$ has a compact shape with no dimension much greater than the mean free path. In this case, $\sigma_{i}$ is constant and $\sigma_{i}$ and $\rho_{i}$ are related by Eq. (38).

\section{SEA equation in diffuse field}

Diffuse field means that energy is homogeneous and isotropic. The question of homogeneity and isotropy can be discussed from Eq. (22). In Eqs. (20), (22) and (30) respectively for energy, intensity and transmitted power, the first term involving $\rho_{i}$ is the direct field whereas the second term involving $\sigma_{i}$ is the reverberant field. In Eq. (22) the constancy of $I_{i}(\mathbf{p}, \varphi)$ requires that $l(\mathbf{r}, \varphi)$ is constant i.e. independent of both $\mathbf{r}$ and $\varphi$ which is impossible for any bounded geometry. In a bounded domain, the direct field is neither homogeneous nor isotropic. Thus, the only chance for the energy and the intensity to be constant is that the direct field is negligible compared with the reverberant field. That is,

$$
\rho_{i} l(\mathbf{r}, \varphi)<<\sigma_{i} .
$$

Assuming that this inequality holds at any point and in any direction, Eqs. (20), (22) and (30) respectively give,

$$
\begin{aligned}
W_{i} & =\frac{\sigma_{i}}{c}, \\
I_{i} & =\frac{\sigma_{i}}{2 \pi}, \\
P_{i \rightarrow j} & =L \frac{\bar{\tau}_{i j}}{\pi} \sigma_{i} .
\end{aligned}
$$

Eqs. (40)-(42) are the fundamental relationships of energy quantities in diffuse field. By eliminating the unknown $\sigma_{i}$, it yields,

$$
\begin{aligned}
I_{i} & =\frac{c_{i}}{2 \pi} W_{i}, \\
P_{i \rightarrow j} & =L \frac{c_{i} \bar{\tau}_{i j}}{\pi} W_{i} .
\end{aligned}
$$


The equality (43), in opposition with Eq. (25) is now valid at any point $\mathbf{r}$ and for any direction $\varphi$. Furthermore, by denoting $S_{i}$ the area of subsystem $i$, the transmitted power is,

$$
P_{i \rightarrow j}=L \frac{c}{\pi S_{i}} \bar{\tau}_{i j} E_{i},
$$

where $E_{i}=W_{i} S_{i}$ is the total vibrational energy of subsystem $i$ with area $S_{i}$. By introducing the coupling loss factor,

$$
\eta_{i j}=L \frac{c \bar{\tau}_{i j}}{\pi \omega S_{i}},
$$

the power transmitted from subsystem $i$ to subsystem $j$ is,

$$
P_{i \rightarrow j}=\eta_{i j} \omega E_{i},
$$

and finally the net exchanged power is,

$$
P_{i j}=\omega\left(\eta_{i j} E_{i}-\eta_{j i} E_{j}\right) .
$$

This is the fundamental equation of SEA. The expression for the coupling loss factor given in Eq. (46) is exactly the same one derived in the conventional wave approach of SEA [10].

The validity domain of SEA is usually evaluated by introducing several parameters. The modal density $n_{i}$ is defined as the number of eigenfrequencies per rad/s,

$$
n_{i}=\frac{S_{i} \omega}{2 \pi c c^{\prime}},
$$

where $c$ is the group speed and $c^{\prime}$ the phase speed. In order to allow a statistical treatment of modes, the mode count $N_{i}$,

$$
N_{i}=n_{i} \Delta \omega>>1,
$$

that is the number of modes within the frequency band $\Delta \omega$, must be large. The modal overlap $M_{i}$ must also be large,

$$
M_{i}=\eta_{i} \omega n_{i}>>1,
$$

where $\eta_{i}$ is the damping loss factor of subsystem $i$. A high modal overlap means that modes overlap each other and therefore that the dynamic behaviour is not dominated by a particular mode. This is the condition for rays to be uncorrelated.

In addition to these two parameters, condition (39) is necessary for direct field to be negligible. By denoting $P_{i}=\rho_{i} S_{i}$ the power being injected in subsystem $i$ and since $\sigma_{i}=c W_{i}=c E_{i} / S_{i}$, Eq. (39) may be re-written as,

$$
P_{i} \frac{\bar{l}}{c}<<E_{i} .
$$

This form of the condition (39) is useful for SEA models since it allows to check a posteriori whether the field is diffuse or not. But, it is also possible to check the condition (39) before SEA equation has been solved, i.e. before $P_{i}$ and $E_{i}$ are known. From Eq. (38), it is apparent that $\rho_{i} \bar{l}$ is much smaller than $\sigma_{i}$ if $\bar{m}_{i} /\left(1-\bar{m}_{i}\right)<<1$ where $\bar{m}_{i}=1-(1-\alpha) \mathrm{e}^{-m l}$. At the first order, for small $\alpha$ and $m \bar{l}$, this is equivalent to $\bar{m}_{i}<<1$ with $\bar{m}_{i}=\alpha+m \bar{l}$. Let us consider the damping loss factor $\eta_{i}$ of subsystem $i$. The energy being dissipated per unit time is $P_{\text {diss }}=\eta_{i} \omega E_{i}$. On the other hand, the energy lost by a single reflection is $\alpha E_{i}$ and since the number of reflections per second is $c / \bar{l}$ the power being absorbed by boundaries is $\alpha c / \bar{l} \times E_{i}$. In addition, the attenuation of 
rays during propagation introduces a further dissipation $m c E_{i}$. The total power being dissipated is thus, $P_{\text {diss }}=\eta_{i} \omega E_{i}=(m c+\alpha c / \bar{l}) \times E_{i}$. The condition $\bar{m}_{i}<<1$ now reads,

$$
\bar{m}_{i}=\frac{\eta_{i} \omega}{c} \bar{l}<<1 .
$$

This last form of the diffuse field condition introduces a dimensionless factor $\bar{m}_{i}$ which is a normalized attenuation factor in opposition with the attenuation factor $m$ which has the dimension of the reciprocal of length. In room acoustics, this factor is called absorption exponent and noted $a^{*}$ in Eq. (5-43) of Ref. [23] whereas in Ref. [24], Eq. (20), it is called the reflectance of plate. The normalized attenuation factor gives the ratio of energy which is absorbed during the propagation to length the mean free path. A ratio greater than one simply means that the energy of rays is totally absorbed in a distance less than the mean free path. A ratio much smaller than one means that the lost of energy of rays is not significant before a large amount of reflections, creating the condition of diffuse field.

The final condition for SEA to apply is that the coupling is weak. Several definitions of the weak coupling can be found in the literature. For instance, in Ref. [24] it is proposed to define the weak coupling of adjacent plates by comparing the mean transmission efficiency $\bar{\tau}_{i j}$ and the reflectance of plate $\bar{m}_{i}$ (the normalized attenuation factor in this text). In the context of the radiative exchanges, it is natural to define the weak coupling as the situation where the transmitted power $P_{i \rightarrow j}=\eta_{i j} \omega E_{i}$ is lower than the dissipated power $\eta_{i} \omega E_{i}$ or, in other words,

$$
\eta_{i j}<<\eta_{i}
$$

WIth Eq. (46) the condition becomes $\bar{\tau}_{i j}<<\pi \omega \eta_{i} S_{i} / c L$ and introducing the normalized attenuation factor $\bar{\tau}_{i j}<<\bar{m}_{i} \pi S_{i} / \bar{l} L$. Finally, Eq. (16) leads to,

$$
\bar{\tau}_{i j}<<\frac{P}{L} \bar{m}_{i} .
$$

This is the definition we adopt in this text for the weak coupling. It is similar to the one of Ref. [24] excepted the presence of the ratio $P / L$ of the perimeter and the coupling length.

These four criteria, mode count $N$, modal overlap $M$, the normalized attenuation factor $\bar{m}$ and the strength of coupling $L \bar{\tau}_{i j} / P \bar{m}$ are all necessary to define the validity domain of SEA. The population of modes $N$ must be large in each sub-system for the statistical method to apply. A modal overlap guarantees that rays are uncorrelated (the condition necessary for energy to be additive) or, in other words, that mode peaks are not too acute. In room acoustics, the so-called Shroeder's frequency $f=2000 \sqrt{(} V / T)$ where $V$ is the room volume and $T$ the reverberation-time is the frequency for which the modal overlap is 3 . Beyond the Shroeder's frequency, modes sufficiently overlap to ensure the validity of the geometrical acoustics approach. The modal overlap is thus related to fluctuations of the actual vibrational level in pure tone compared with the mean value in broadband predicted by SEA. The necessity of the third criterion, the normalized attenuation factor $\bar{m}$, clearly appears in the proof of SEA equations presented in this section. The condition $\bar{m}<1$ ensures that the vibrational field is diffuse that is each sub-system is in thermal equilibrium. Finally a light coupling means that two coupled sub-systems can be individually in thermal equilibrium but in the mean time, they are not jointly in thermal equilibrium and therefore they can exchange a vibrational power. 
(a)

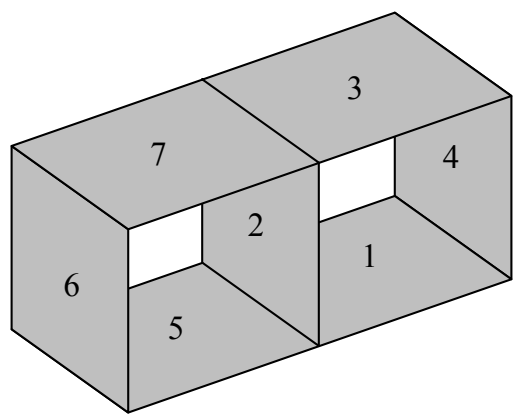

(b)

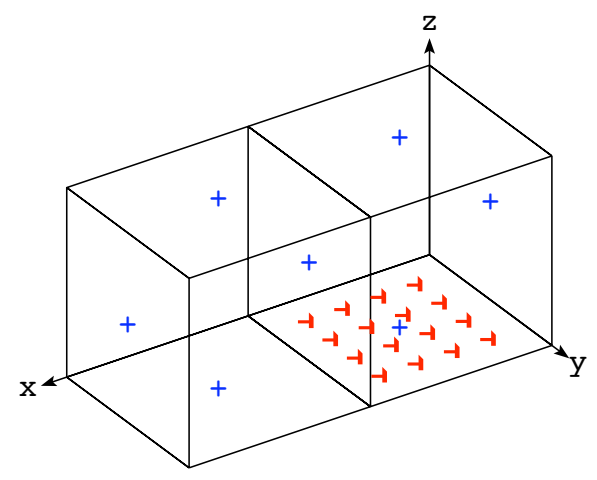

Figure 3: Geometry of the seven plates structure. (a), General view. (b), Position of the driving points (small hammers) and the receiver points $(+)$.

\section{$5 \quad$ Numerical simulation}

In order to test the validity of previous theoretical developments, a numerical simulation is proposed on the structure shown in Fig. 3a. This structure is made of seven square plates. All plates are identical and are made of aluminium, volumic mass $\rho=2700 \mathrm{~kg} / \mathrm{m}^{3}$, Young's modulus $E=71 \mathrm{GPa}$, Poisson's coefficient $\nu=0.3$, size $L=1 \mathrm{~m}$ and thickness $h=1 \mathrm{~mm}$.

The assembly is realized with four L-junctions and two T-junctions. The junctions are assumed to be massless and infinitely rigid. The transmission and reflection efficiencies are calculated on the basis of the continuity of displacements and rotations and the equilibrium of forces and moments. All details are available in Refs. [8,9]. All uncoupled edges of plates are assumed to be simply supported. This condition is indeed chosen in order to simplify the reference calculation (Appendix).

\begin{tabular}{lllll}
\hline & $1 \mathrm{kHz}$ & $2 \mathrm{kHz}$ & $4 \mathrm{kHz}$ & $8 \mathrm{kHz}$ \\
\hline$l / \lambda$ & 8 & 11 & 15 & 22 \\
$N$ & 303 & 607 & 1215 & 2430 \\
$\bar{\tau}_{14}$ & 0.33 & 0.32 & 0.32 & 0.32 \\
$\bar{\tau}_{12}$ & 0.15 & 0.15 & 0.15 & 0.15 \\
$\bar{\tau}_{15}$ & 0.15 & 0.14 & 0.13 & 0.13 \\
\hline
\end{tabular}

Table 1: Number of wavelengths $\bar{l} / \lambda$, mode count $N$ and mean efficiencies $\bar{\tau}_{i j}$ for L- and T-junctions.

The wavelength is about $10 \mathrm{~cm}$ at $1 \mathrm{kHz}$ and $4 \mathrm{~cm}$ at $8 \mathrm{kHz}$ for the bending wave. This is large enough compared with the thickness to apply the Love plate equation. Tab. 1 summarizes the number of wavelengths in the mean free path from $1 \mathrm{kHz}$ to $8 \mathrm{kHz}$ which is always greater than eight. This value ensures that a high frequency model makes sense. The modal density from Eq. (49) is constant and has the value 0.048 meaning that $2 \pi \times 1000 \times n=303$ modes are included within the octave band centred on $1 \mathrm{kHz}$ and more for other octave bands. This is an assessment of the population of modes we are dealing with. 


\begin{tabular}{l|llll}
\hline $\mathrm{M}$ & $1 \mathrm{kHz}$ & $2 \mathrm{kHz}$ & $4 \mathrm{kHz}$ & $8 \mathrm{kHz}$ \\
\hline$\eta=0.1 \%$ & 0.3 & 0.6 & 1.2 & 2.4 \\
$\eta=1 \%$ & 3 & 6 & 12 & 24 \\
$\eta=10 \%$ & 30 & 60 & 120 & 240 \\
\hline
\end{tabular}

Table 2: Modal overlap $M$ versus frequency and damping loss factor.

\begin{tabular}{l|llll}
\hline $\bar{m}$ & $1 \mathrm{kHz}$ & $2 \mathrm{kHz}$ & $4 \mathrm{kHz}$ & $8 \mathrm{kHz}$ \\
\hline$\eta=0.1 \%$ & 0.02 & 0.03 & 0.05 & 0.07 \\
$\eta=1 \%$ & 0.2 & 0.3 & 0.5 & 0.7 \\
$\eta=10 \%$ & 2 & 3 & 5 & 7 \\
\hline
\end{tabular}

Table 3: Normalized attenuation factor $\bar{m}$ versus frequency and damping loss factor.

The modal overlaps are summarized in Tab. 2. It can be seen that the condition of Eq. (51) is fulfilled when $\eta \geq 1 \%$. Beyond this value, modes are expected to sufficiently overlap to be lost in the frequency average. This condition is necessary for both, SEA and non-diffuse equations of Section 2 to apply. The normalized attenuation factors are summarized in Tab. 3. The condition of Eq. (53) is fulfilled when $\eta \leq 1 \%$. This is the additional condition for SEA to apply. Thus, it is expected that SEA gives correct results in the only case $\eta=1 \%$. When $\eta=10 \%$ the modal overlap is large enough meaning that no mode dominates the dynamics. But $\bar{m}>1$ and therefore the field is not diffuse. When $\eta=0.1 \%$, the normalized attenuation factor is correct but not the modal overlap.

The mean efficiencies are about 0.13 for the T-junction and 0.3 for the L-junction. With a ratio $P / L=2$, the coupling is weak (Eq. (55)) for $\eta=1 \%$ and $\eta=10 \%\left(\bar{\tau}_{i j}<2 \bar{m}_{i}\right)$ but the coupling is strong for $\eta=0.1 \%\left(\bar{\tau}_{i j}>2 \bar{m}_{i}\right)$. This result also holds when adopting the definition of Ref. [24].

Plate 1 (bottom right) is excited by sixteen driving points located at $x=0.2,0.4,0.6,0.8$ and $y=0.2,0.4,0.6,0.8$ in the frame of Fig. 3. The receiver points are located at the centre of each plate. Each driving point applies a force of $F=1 \mathrm{~N}$ (peak value). The resulting injected power is assessed with the impedance of infinite plates, $P_{\text {inj }}=|F|^{2} / 16 \sqrt{D \rho h}=14.8 \mathrm{~mW}$ where $D$ is the bending stiffness of the plate. This relationship shows that the injected power does not depend on the frequency neither the damping loss factor.

Three calculations have been done for each octave band (1 to $8 \mathrm{kHz}$ ) and each damping loss factor $(\eta=0.1 \%, 1 \%$ and $10 \%)$. The first one is a SEA calculation with the coupling loss factors given in Eq. (46). Indeed some other more elaborated coupling loss factors are available in the literature [24]. However, the purpose is to demonstrate the convergence of radiative exchange equations to SEA in same conditions rather than to test SEA itself. This is the motivation of this choice. The equation of SEA of this system is a linear set of seven equations. The second calculation is based on equations of Section 2. The system of Eqs. (8), (10) is first solved by using the collocation method with sources being constant on the segments. Once the unknowns $\sigma_{i}$ have been determined, the energy density is computed with Eq. (1). The description of this algorithm is available in Ref. [25]. For this system, the boundary is discretized with 322 boundary elements that is more than 10 elements per edge of plate. The third calculation is the reference one. It is based on a semi-analytical solution of the governing equation of a Love plate. The deflection is developed in a Fourier series along the $y$-axis. The simply supported conditions are then fulfilled. 
(a)
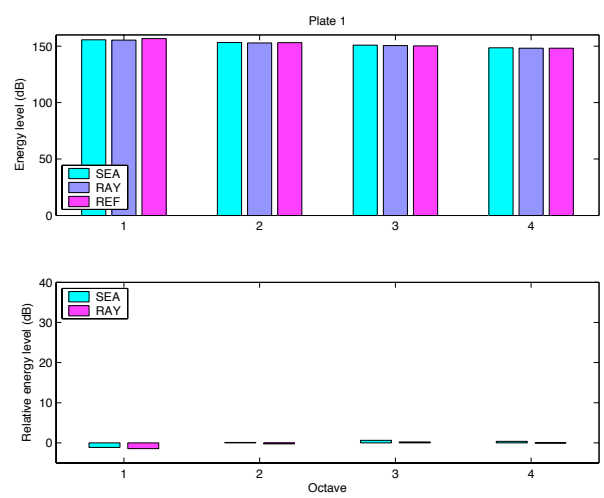

(c)
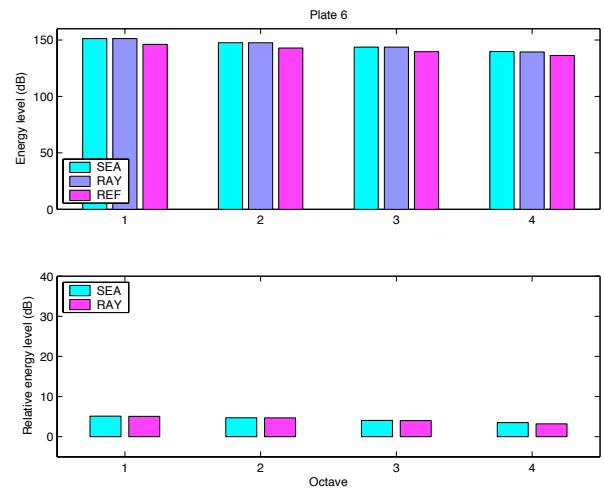

(b)
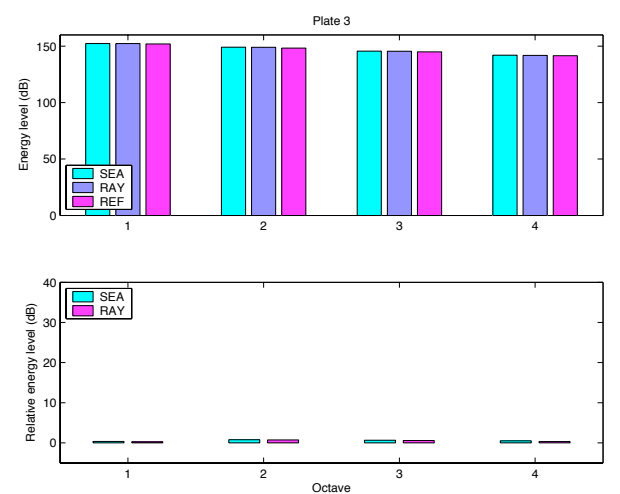

(d)
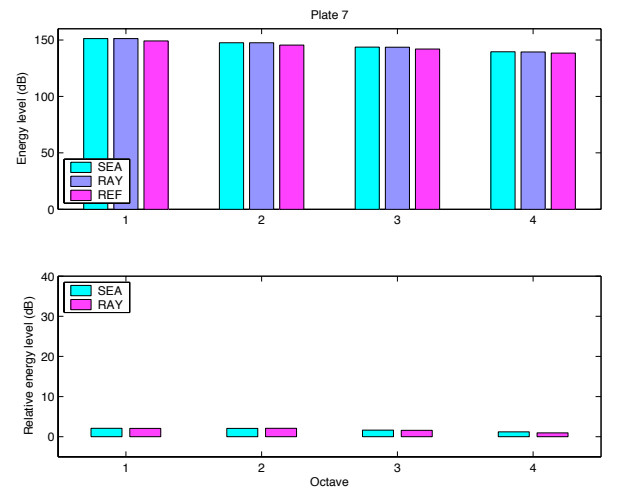

Figure 4: Vibrational response of (a), plate 1; (b), plate 3; (c), plate 6; (d), plate 7 for a damping loss factor $\eta=0.1 \%$. 
(a)
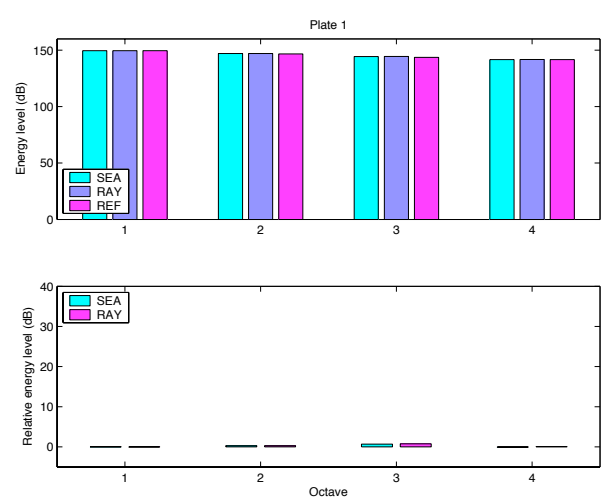

(c)
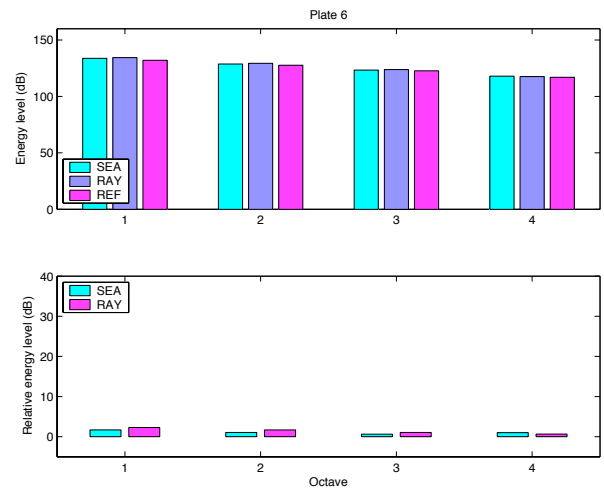

(b)
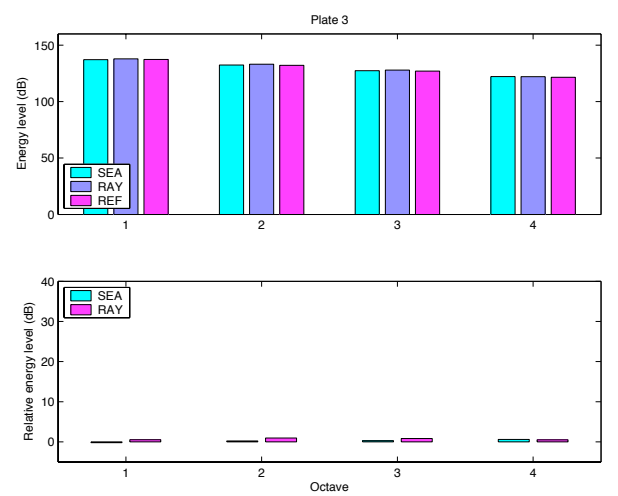

(d)
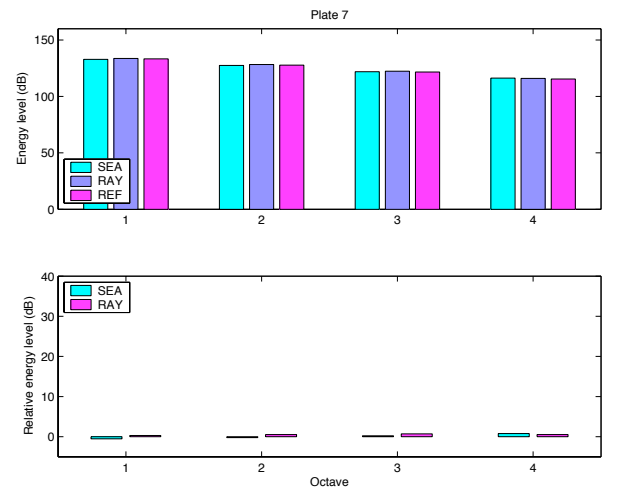

Figure 5: Vibrational response of (a), plate 1; (b), plate 3; (c), plate 6; (d), plate 7 for a damping loss factor $\eta=1 \%$. 
(a)
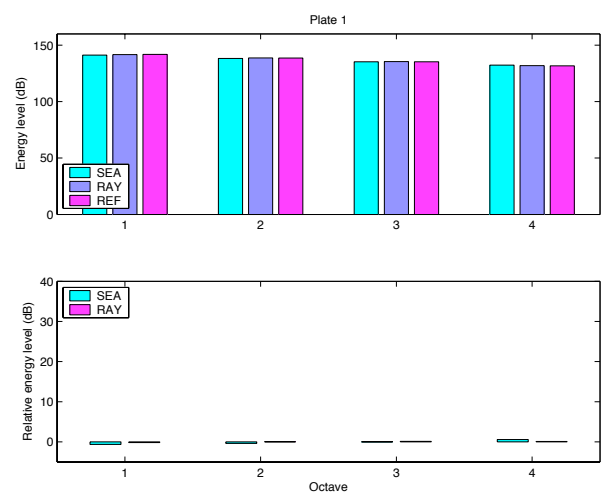

(c)
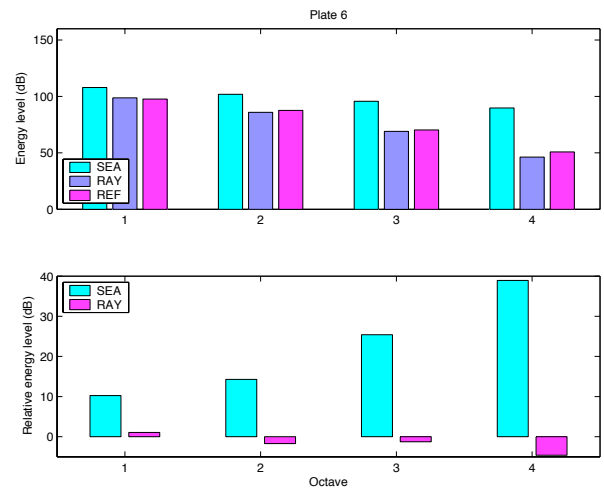

(b)
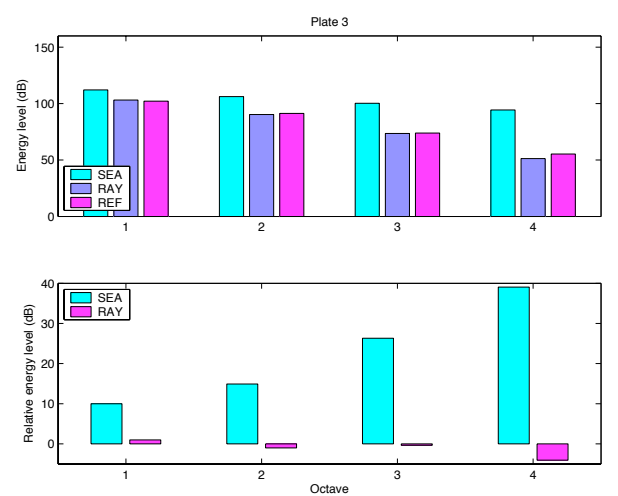

(d)
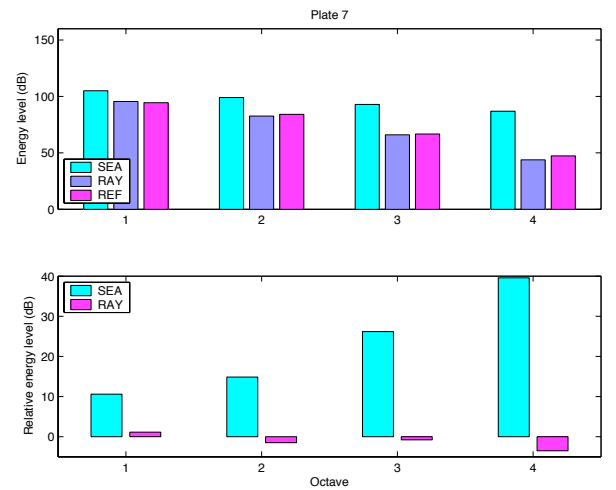

Figure 6: Vibrational response of (a), plate 1; (b), plate 3; (c), plate 6; (d), plate 7 for a damping loss factor $\eta=10 \%$. 
Along the $x$-axis (or $z$-axis for vertical plates), the deflection is the sum of four travelling waves. The total number of unknowns coefficients is thus 28. The coupling conditions at both edges of the plates give 4 equations for a L-junction and 6 equations for a T-junction giving the 28 expected conditions (Appendix). This calculation is done from $707 \mathrm{~Hz}$ up to $11312 \mathrm{~Hz}$ (4 octaves), one step per Hertz, and for the 16 loading cases. The final result on each plate is the RMS-response over each octave band, and the sum of the 16 loading cases assuming to be uncorrelated.

Figs. 4-6 show the energy density for plates 1, 3, 6 and 7 versus octave band for each damping loss factor. It can be observed that both, SEA and radiative exchanges lead to similar results when $\eta=0.1 \%$ and $\eta=1 \%$. In these two cases, the normalized attenuation factor $\bar{m}$ is less than unity (Tab. 3) showing that the energy field is diffuse. However, an important discrepancy can be seen for the case $\eta=10 \%$. The differences can reach up to $40 \mathrm{~dB}$ for plate 6 which is the most distant plate from driving points. In this case, the normalized attenuation factor is greater than unity showing that the field is not diffuse and thus, an important assumption of SEA is violated. On the other hand, the reference results show that for $\eta=0.1 \%$ some slight differences may appear with the energy methods. This is the case when the modal overlap (Tab. 2) is low. However, the agreement between the reference calculation and both energy methods is quite good for $\eta=1 \%$ for which the modal overlap is high and the normalized attenuation factor is low. When $\eta=10 \%$, reference calculation and radiative exchanges are still in good agreement, but not with SEA, showing that the validity domain of radiative exchange equations is larger than the one of SEA.

\section{Diagram of validity}

The validity domain of the energy methods may be assessed. Both methods require to have a large number of modes $N$. But, the modal density $n$ is constant in frequency for plates and since the bandwidth is doubled for successive octave bands, it yields $N \propto f$ where $f$ is the central frequency. The limit $N=100$ is then a vertical line in the $\eta, f$-plane of Fig. 7. According to Eq. (51), $M \propto \eta f$. The boundary of the domain $M>1$ is therefore the hyperbolic line $\eta=1 / 2 \pi n f$ which is the bottom solid line of Fig. 7. Finally, the group speed $c$ is proportional to the square root of the frequency. From Eq. (53), it results in $\bar{m} \propto \eta \sqrt{f}$. The upper solid line of Fig. 7 is therefore $\eta=a / \sqrt{f}$ where $a$ is a proportionality constant. The validity domain of the energy exchanges is $N>100$ and $M>1$ and is thus quarter plane shown in Fig. 7. The validity domain of SEA is limited by $N>100, M>1$ and $\bar{m}<1$ and is therefore the half-strip shown in Fig. 7. The only case located within the strip is $\eta=1 \%$ where the three methods well agree.

\section{Conclusion}

In conclusion, the present paper has shown that the radiative exchange equations in structural ray theory converge to SEA equations when the field becomes diffuse. The coupling loss factor derived by this convergence process matches with the simplest one commonly derived in SEA literature and the well-known proportionality of exchanged power with the difference of energy densities is recovered.

The numerical simulations proposed in this paper highlight how it is important to check the validity domain of a method before to use it. Diagrams such as the one presented in Section 6 can be useful to this end. Validity of SEA and the ray method is confined within a domain defined 


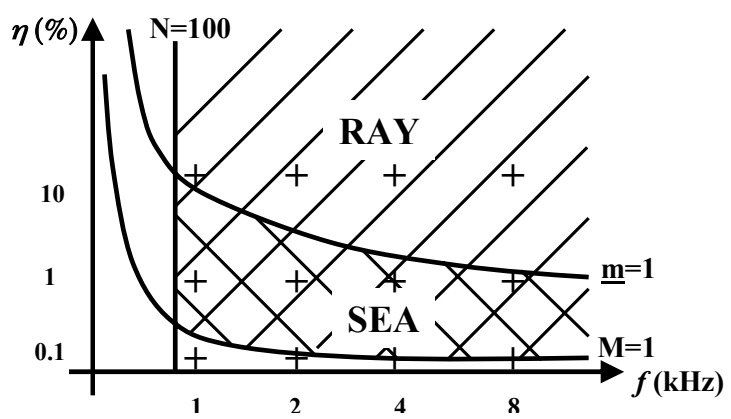

Figure 7: Validity domain SEA and uncorrelated rays in frequency - damping loss factor plane. $(\backslash \backslash)$, domain of SEA defined by $N>100, M>1$ and $\bar{m}<1$; $(/ /)$, domain of rays defined by $N>100$ and $M>1 ;(+)$, position of the twelve calculations of Figs. 4-6.

by three boundary curves in the $f, \eta$-plane (constant mode count, modal overlap and normalized attenuation factor). The two first conditions delimit the validity of the ray method whereas the three conditions are necessary for SEA. To use SEA or the ray method on the left side of $N=$ cste is simply meaningless. Both methods require to be in the high frequency range. This is a shortcoming commonly encountered in SEA models that a sub-system has a too low mode count for instance, when a sub-system is stiffer than the adjacent sub-systems. This difficulty is the main motivation for the studies on the coupling of the finite element method with SEA. Below the curve $M=$ cste is the domain of large fluctuations. SEA and the ray method apply in principle but are inaccurate. Their use is therefore unwise. A low modal overlap is encountered in undamped structures made of metal for instance. A dominating mode within a single sub-system can affect not only the structural response of this sub-system but also the transmission through this sub-system. The entire system can therefore be affected. The prediction of fluctuations around the mean value of SEA is a crucial question in this regard. This has inspired many studies on confidence and SEA. The line $\bar{m}=$ cste is the boundary between SEA and the ray method. To increase the damping loss factor leads to a non-diffuse field which can damage SEA results. But, this is also the case by increasing the frequency. This fact is inconsistent with the widely spread idea that SEA results are better as the frequency increases. SEA is valid in high frequencies indeed, but provided that the field remains diffuse. From this point of view, the normalized attenuation factor appears to be an appropriate criterion of the validity of the diffuse field assumption. The knowledge of this limit is obviously useful for SEA but also for the ray method. In principle, the ray method applies in diffuse field but its use instead of SEA would be inappropriate since it requires more computation than SEA.

\section{Appendix A}

The reference calculation for the structure shown in Fig 3 is considered in this Appendix. As stated in Section 5, Love's equation $\Delta^{2} v-k^{4} v=F_{0} / D \times \delta\left(x-x_{0}\right) \delta\left(y-y_{0}\right)$ applies for the transverse deflection $v(x, y)$. $D$ is the bending stiffness of the plate, $F_{0}$ the transverse force at frequency $\omega$ applied to point $x_{0}, y_{0}$ and $k$ the wavenumber at same frequency. For each plate $i$, the transverse 
deflection $v_{i}$ is developped in a Fourier series,

$$
v_{i}(x, y)=\sum_{n=1}^{\infty} v_{i, n}(x) \sin \left(\frac{n \pi y}{L}\right),
$$

The boundary conditions along the simply supported edges $y=0$ and $y=L$ are satisfied. Introducing the Fourier's series into Love's equation leads to a fourth order differential equation for each Fourier's term $v_{i, n}$ whose solution is,

$$
\begin{aligned}
v_{i, n}(x)= & A_{i, n} \exp ^{-\beta_{n} x}+B_{i, n} \exp ^{-\beta_{n}(L-x)}+C_{i, n} \exp ^{-\gamma_{n} x}+D_{i, n} \exp ^{-\gamma_{n}(L-x)}+ \\
& E_{i, n} \exp ^{-\beta_{n}\left|x_{0}-x\right|}+F_{i, n} \exp ^{-\gamma_{n}\left|x_{0}-x\right|} .
\end{aligned}
$$

The four first terms in the right-hand side are the solution of the homogeneous fourth order differential equation and $\beta_{n},-\beta_{n}, \gamma_{n},-\gamma_{n}$ are the four roots of its characteristic equation,

$$
X^{4}-2\left(\frac{n \pi}{L}\right)^{2} X^{2}-\left[k^{4}-\left(\frac{n \pi}{L}\right)^{4}\right]=0 .
$$

The two last terms in the right-hand side of Eq. (57) are proportional to the fundamental solution of the fourth order differential equation with the conditions $E_{i, n} \beta_{n}+F_{i, n} \gamma_{n}=0$ and $E_{i, n} \beta_{n}^{3}+F_{i, n} \gamma_{n}^{3}=$ $-F_{0} / L D \times \sin \left(n \pi y_{0} / L\right)$.

The constants $A_{i, n}, B_{i, n}, C_{i, n}$ and $D_{i, n}$ are determined by applying the coupling conditions at the interfaces $x=0$ and $x=L$. For the L-coupling between subsystems 5 and 6 of Fig. 3, these conditions are,

$$
\begin{aligned}
v_{5, n}(L) & =0, \\
v_{6, n}(0) & =0, \\
\partial_{x} v_{5, n}(L) & =\partial_{x} v_{6, n}(0), \\
D_{5}\left[\partial_{x}^{2} v_{5, n}(L)+\nu \partial_{y}^{2} v_{5, n}(L)\right] & =D_{6}\left[\partial_{x}^{2} v_{6, n}(0)+\nu \partial_{y}^{2} v_{6, n}(0)\right],
\end{aligned}
$$

where due to the Fourier's series development, $\partial_{y}^{2}$ denotes a multiplication by $-(n \pi / L)^{2}$ and $D_{i}$ is the bending stiffness of plate $i$. While for the T-coupling 1-2-5 of Fig. 3, the conditions are,

$$
\begin{aligned}
v_{1, n}(L)= & 0 \\
v_{2, n}(0)= & 0 \\
v_{5, n}(0)= & 0 \\
\partial_{x} v_{1, n}(L)= & \partial_{x} v_{2, n}(0), \\
\partial_{x} v_{1, n}(L)= & \partial_{x} v_{5, n}(0), \\
D_{1}\left[\partial_{x}^{2} v_{1, n}(L)+\nu \partial_{y}^{2} v_{1, n}(L)\right]= & D_{2}\left[\partial_{x}^{2} v_{2, n}(0)+\nu \partial_{y}^{2} v_{2, n}(0)\right]+ \\
& D_{5}\left[\partial_{x}^{2} v_{5, n}(0)+\nu \partial_{y}^{2} v_{5, n}(0)\right] .
\end{aligned}
$$

Finally, the energy density density at any receiver point is given by,

$$
W(x, y)=\frac{\rho \omega^{2}}{4}|v|^{2}+\frac{D}{4}\left[\left|\frac{\partial^{2} v}{\partial x^{2}}\right|^{2}+\left|\frac{\partial^{2} v}{\partial y^{2}}\right|^{2}+2 \nu \operatorname{Re}\left(\frac{\partial^{2} v}{\partial x^{2}} \frac{\partial^{2} v^{*}}{\partial y^{2}}\right)+2(1-\nu)\left|\frac{\partial^{2} v}{\partial x \partial y}\right|^{2}\right],
$$

where Re designates the real value, ${ }^{*}$ the complex conjugate and $\rho$ the mass per unit area. 


\section{References}

[1] R.H. Lyon and G. Maidanik. Power flow between linearly coupled oscillators. Journal of the Acoustical Society of America, 34:623-639, 1962.

[2] D.E. Newland. Calculation of power flow between coupled oscillators. Journal of Sound and Vibration, 3:262-276, 1966.

[3] T.D. Scharton and R.H. Lyon. Power flow and energy sharing in random vibration. Journal of the Acoustical Society of America, 43:1332-1343, 1968.

[4] A. Carcaterra. An entropy formulation for the analysis of energy flow between mechanical resonators. Mechanical Systems and Signal Processing, 16:905-920, 2002.

[5] B. Mace. Statistical energy analysis: coupling loss factors, indirect coupling and system modes. Journal of Sound and Vibration, 279:141-170, 2005.

[6] G. Maidanik and J. Dickey. Wave derivation of the energetics of driven coupled onedimensional dynamic systems. Journal of Sound and Vibration, 139:31-42, 1990.

[7] B. Mace. Power flow between two continuous one-dimensional subsystems: a wave solution. Journal of Sound and Vibration, 154:289-319, 1992.

[8] Beckmann Th. Wöhle W. and Schreckenbach H. Coupling loss factors for statistical energy analysis of sound transmission at rectangular slab joints, part. I. Journal of Sound and Vibration, $77(3): 323-334,1981$.

[9] Beckmann Th. Wöhle W. and Schreckenbach H. Coupling loss factors for statistical energy analysis of sound transmission at rectangular slab joints, part. II. Journal of Sound and Vibration, 77(3):335-344, 1981.

[10] R.H. Lyon. Statistical Energy Analysis of dynamical systems. Cambridge, Massachusetts, MIT Press, 1975.

[11] L. Cremer, M. Heckl, and E.E. Ungar. Structure-Borne Sound: Structural Vibrations and Sound Radiation at Audio Frequencies. Springer, 1990.

[12] A.J. Keane and W.G. Price. Statistical Energy Analysis, an overview with applications in structural dynamics. Cambridge, university press, 1997.

[13] F. Fahy. Statistical energy analysis: a critical overview. Philosophical Transactions of the Royal Society of London, A346:431-447, 1994.

[14] R.S. Langley. A wave intensity technique for the analysis of high frequency vibrations. Journal of Sound and Vibration, 159:483-502, 1992.

[15] O.M. Bouthier and R.J. Bernhard. Simple models of energy flow in vibrating plates. Journal of Sound and Vibration, 182:149-164, 1995.

[16] A. Le Bot. Energy transfer for high frequencies in built-up structures. Journal of Sound and Vibration, 250(2):247-275, 2002.

[17] M.F. Modest. Radiative heat transfer. Mc Graw-Hill Inc., 1993.

[18] Kuttruff H. Simulierte nachhallkurven in rechteckräumen mit diffusem schallfeld. Acoustica, 25:333-342, 1971. 
[19] L. Maxit and J.L. Guyader. Extension of sea model to subsystems with non-uniform modal energy distribution. Journal of Sound and Vibration, 265(2):337-358, 2003.

[20] A. Le Bot. Comparison of vibrational conductivity and radiative energy transfer methods. Journal of Sound and Vibration, 283:135-151, 2005.

[21] A. Le Bot and A. Bocquillet. Comparison of an integral equation on energy and the ray-tracing technique for room acoustics. J. Acoust. Soc. Am., 108:1732-1740, 2000.

[22] V.I. Arnold and A. Avez. Problèmes ergodiques de la mécanique classique. Gauthier-Villars, 1967.

[23] H. Kuttruff. Room acoustics. London, Spon Press, 4 edition, 2000.

[24] E.C.N. Webster and B. Mace. Statistical energy analysis of two edge-coupled rectangular plates: ensemble averages. Journal of Sound and Vibration, 193:793-822, 1996.

[25] A. Le Bot. A vibroacoustic model for high frequency analysis. Journal of Sound and Vibration, 211(4):537-554, 1998 